\title{
Upping Recruitment in Clinical Trials: Are the Costs Worth It?
}

\author{
June M. McKoy Athena T. Samaras Thanh H. Luu Charles L. Bennett \\ Department of Medicine, Divisions of Hematology/Oncology and Geriatric Medicine and Robert H. Lurie Comprehensive Cancer Center, \\ Northwestern University Feinberg School of Medicine, Chicago, Illinois
}

Special challenges are frequently encountered when trying to increase patient recruitment into clinical trials. In clinical trials, it is necessary that large numbers of patients are recruited to allow for subject stratification for large variables, thereby contributing greatly to slow progress in reaching recruitment targets. While clinical trials are critical to improving patient outcomes in all areas, including cancer, strategy modifications are essential to balance recruitment efforts against trial costs. The allocation of scarce health care resources is increasingly based upon economic evaluations of health care interventions. Since clinical trials have the potential to dissipate many of these resources they must be conducted with costs in mind.

In the current issue of ONKOLOGIE, Jacobs reports on the pressures faced by clinicians to increase patient participation in clinical trials and the attendant financial impact on medical institutions that choose to do so [1]. Concerned about the difficulties inherent in separating medical care from 'study care' for patients enrolled in clinical trials, the author postulates that clinical trial costs should be reimbursed in concert with standard of care cancer costs. He utilizes a decision-making algorithmic process to determine participation in clinical trials based on economic and budget impacts. The economic model presented was derived from both prospective and retrospective studies evaluating the procedures and costs associated with clinical trial protocols for breast and ovarian cancer. Procedures and cost estimates were obtained from consultations with a center that manages clinical trials and with several hospital departments.

Jacobs found that, to control for clinical trial costs, a network of providers with expertise in economics is needed; costs should be summed from individual costs of each service provided based on a detailed research protocol. The author also found that costs must be separated by those that are directed towards medically-related interventions and those that are solely related to the study, presumably at the physician's discretion. Additionally, the author postulated that this cost separation is necessary since sponsors, rather than institutions, should cover sponsor-related costs; presently some of these costs are borne by physicians and medical centers.

The author also proffers the use of marginal cost estimation of services as calculated based on averaging flat rates to assist in determining cost allocation. Jacobs next recommends a series of steps designed to further delineate costs prior to pursuing a contractual relationship with a study sponsor. For these steps, Jacobs proposes the evaluation of the study contract to determine depth of reimbursement and allocation of off-label medications. His model urges physicians to make a concerted effort to renegotiate contracts that fail the above costs tests and where negotiations fail, to redirect these efforts towards study protocol revisions that are in line with existing cost structures at the medical institution. Institutions and physicians are also encouraged to underwrite observational study costs or refer patients to community institutions with better cost structures and comparable cancer therapies.

There are some practical questions that arise in interpreting these findings and recommendations. What is the clinical impact of demonstrating separated costs in the context of clinical trials? For trials that have the potential to prolong survival and improve quality of life, costs negotiations could insert negative outcomes into the equation. Furthermore, clinical trial costs may also be affected by factors that are not mediated through the trial and by mechanisms that are independent of the trial process. Some of these factors include unexpected opportunity costs and costs related to adverse drug reactions or events. Regulatory mandates and subject response to therapies might also lead to prolongation of trials and increase in costs.

In our prior pilot study that investigated clinical trial costs we also found that detailed economic information was criti-

\section{KARGER}

Fax +497614520714

Information@Karger.de

www.karger.com (c) 2009 S. Karger GmbH, Freiburg

Accessible online at:

www.karger.com/onk
Charles L. Bennett, MD

Northwestern University Feinberg School of Medicine

Division of Hematology/Oncology

710 N Fairbanks Ct, \#8256, Chicago, IL 60611, USA

Tel. +1 312 503-0804, Fax -1040

cbenne@northwestern.edu 
cal to the analyses [2]. Our study of 70 cancer patients enrolled in phase II clinical trials found that, in 1996, charges for participants on cancer-related clinical trials were no greater than charges for participants incurred outside of the clinical trial setting. These estimates were comparable with those reported from other single-site studies [3, 4]. Furthermore, in their study measuring the cost-effectiveness of seven selected randomized trials, Detsky et al. found these trials to be good investments, with lower dollar per life-year saved ratios than those associated with the proven interventions [5].

Another study by Chirikos et al. supports the findings from prior studies that revealed no significant increase in treatment costs for cancer patients enrolled in clinical trials [6]. The study, however, had notable limitations: it excluded physician fees, looking only at in-patient and out-patient hospital care; it used data on charges from hospital billing records, which do not comport well with actual payments and underlying re- source costs; costs were adjusted for the type and complexity of the initial therapy, potentially underestimating trial associated costs [6]. Unquestionably, overall costs for clinical trials have increased with time and many of these studies should be revisited to reassess the findings.

The model proposed in this article, though transparent and seemingly straight-forward, should be revised or revisited to take into account other cost criteria of relevance. Until then, this model should be utilized against the backdrop of the limitations mentioned previously.

\section{Conflict of Interest}

This project is funded in part by Grant No. 1K01CA134554-01 from the National Cancer Institute (J.M.M) and the Research on Adverse Drug events And Reports (RADAR) Project. The authors have no conflict of interest.

\section{References}

1 Jacobs VR: Making or losing money with participation in clinical trials: a decision analysis. Onkologie 2009;32:411-416.

2 Bennett CL, Adams JR, Knox KS, Kelahan AM, Silver SM, Bailes JS: Clinical trials: are they a good buy? J Clin Oncol 2001;19:4330-4339.
3 Wagner JL, Alberts SR, Sloan JA, Cha S, Killian $\mathrm{J}$, O'Connell MJ, van Grevenhof P, Lindman J, Chute CG: Incremental costs of enrolling patients in clinical trials: a population based study. J Natl Cancer Inst 1999;91:847-853.

4 Fireman BH, Fehrenbacher L, Gruskin EP, et al: Cost of care for patients in cancer clinical trials. J Natl Cancer Inst 2000;92:136-142.
5 Detsky AS: Are clinical trials a cost-effective investment? JAMA 1989;262:1795-1800.

6 Chirikos TN, Ruckdeschel JC, Krischer JP: Impact of clinical trials on the cost of cancer care. Med Care 2001;39:373-383. 